\title{
Lack of association between the FAS/FASL polymorphisms and cervical cancer risk: A meta-analysis
}

\author{
YINGYING DU*, LIXIA HU* and YUEYIN PAN \\ Department of Oncology, The First Affiliated Hospital of Anhui Medical University, Hefei, Anhui 230022, P.R. China
}

Received November 30, 2012; Accepted January 11, 2013

DOI: 10.3892/br.2013.56

\begin{abstract}
FAS/FASL gene promoter polymorphisms are associated with cervical cancer risk, however, results from previous studies have been conflicting. To obtain a more precise estimation of the association between these polymorphisms and cancer risk, a meta-analysis was performed. All eligible studies up to November 1st, 2012, concerning FAS-670 A/G, FAS-1377 G/A and FASL-844 T/C polymorphisms and cervical cancer risk, were collected from the following electronic databases: PubMed, Excerpta Medica Database and Chinese Biomedical Literature Database. The odds ratio (OR) and $95 \%$ confidence interval $(95 \% \mathrm{CI})$ were used to assess the strength of the association via the additive, codominant, dominant and recessive models. In total, 10 publications with 11 case-control studies (10 on FAS-670 A/G, 5 on FAS-1377 G/A and 6 on FASL-844 T/C polymorphisms) were included in this meta-analysis. No association between FAS-670 A/G, FAS-1377 G/A and FASL-844 T/C polymorphisms and cervical cancer susceptibility for all the genetic models was identified. Following stratification of the studies by ethnicity or source of controls, similar results were obtained. In conclusion, our findings showed that the FAS-670 A/G, FAS-1377 G/A and FASL-844 T/C polymorphisms are not associated with cervical cancer risk. Future studies with larger sample sizes are required to further evaluate these associations.
\end{abstract}

\section{Introduction}

Cervical cancer was the third most commonly diagnosed cancer and the fourth leading cause of cancer death in females worldwide in 2008 (1). Risk factors for cervical cancer include early onset of sexual activity, multiple sexual

Correspondence to: Professor Yueyin Pan, Department of Oncology, The First Affiliated Hospital of Anhui Medical University, 218 Ji Xi Road, Hefei, Anhui 230022, P.R. China

E-mail: yueyinpan1965@gmail.com

*Contributed equally

Key words: cervical cancer, FAS, FASL, polymorphism, meta-analysis partners, cigarette smoking, high parity, low socioeconomic status, immunosuppression, high-risk sexual partners (2) and particularly, human papillomavirus (HPV) infection (3). HPV infections are widespread in the general population. However, only a small proportion of infected women progress to cervical cancer (4), suggesting that the development of cervical cancer may be influenced by genetic factors.

FAS (also known as Apo-1 or CD95), a cell-surface receptor, plays a key role in apoptotic signaling in many cell types (5). FAS ligand (FASL) (also known as CD95L), a member of the tumor necrosis factor super family, can trigger an apoptotic cascade by cross-linking with its receptor, FAS $(6,7)$. Single nucleotide polymorphisms in the promoter regions of FAS and FASL have been associated with the differential expression of these two genes. It was reported that a $G \rightarrow A$ transition at position -1377 and an $A \rightarrow G$ transition at position -670 in the promoter region of the FAS gene destroys stimulatory protein (Sp) 1 and signal transducer and activator of transcription (STAT) 1 protein-binding element, reducing promoter activity and decreasing FAS expression $(8,9)$. In regards to the FASL gene, it has been shown that a $\mathrm{T} \rightarrow \mathrm{C}$ transition at position -844 in a binding motif for CAAT/ enhancer-binding protein $\beta$ induces a significantly higher basal expression of FASL (10).

Although evidence of an association between FAS/FASL polymorphisms and cervical cancer risk has been reported, the findings remain controversial (11-22). Therefore, we performed a meta-analysis of all published studies on the association between the FAS/FASL polymorphisms and cervical cancer.

\section{Materials and methods}

Publication search. A search of the literature was performed using PubMed, Excerpta Medica Database and the Chinese Biomedical Literature Database to identify articles that evaluated the associations between polymorphisms in FAS/FASL and cervical cancer risk (last search was updated on November 1st, 2012). The search terms used were: 'FAS or CD95 or FASL or CD95L', 'cervical carcinoma or cervical cancer or cervix cancer' and 'polymorphism or polymorphisms'. In addition, we checked all the references of relevant reviews and eligible articles that our search retrieved. No language restrictions were applied. If more than one article was published by the same author using the same case series, we selected the research with the largest sample size. 

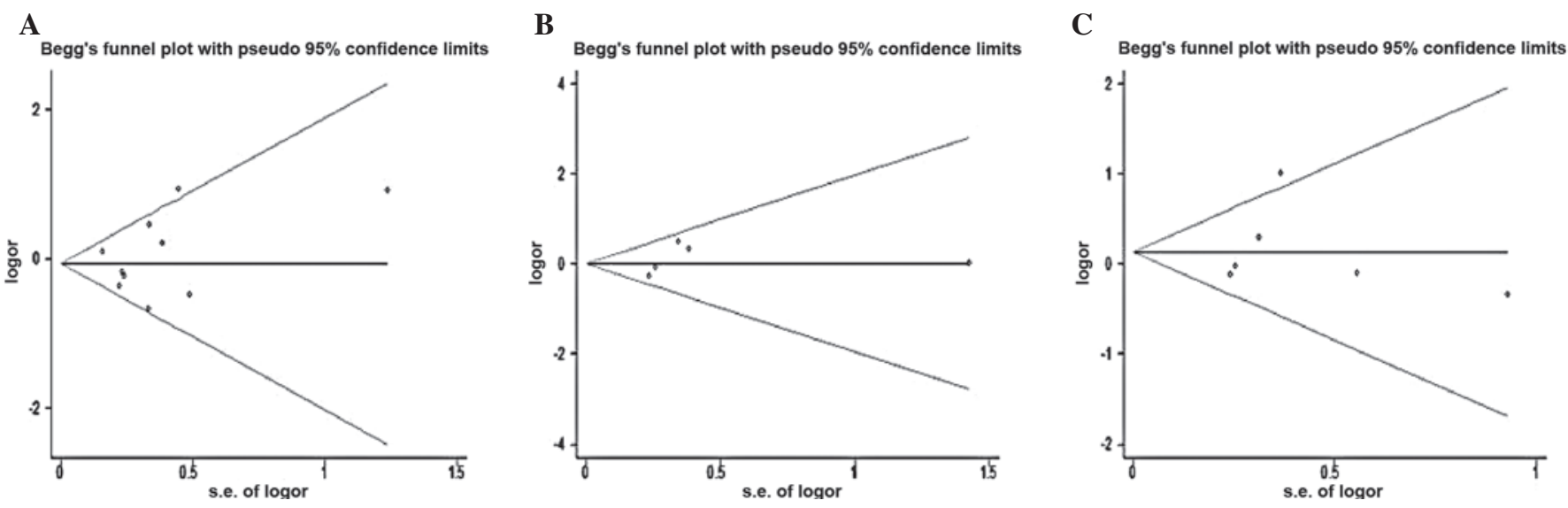

Figure 1. Begg's funnel plot indicated that no publication bias was observed (A) for GG vs. AA comparison in FAS-670 A/G polymorphism; (B) for AA vs. GG comparison in FAS-1377G/A polymorphism; (C) for CC vs. TT comparison in FASL -844T/C polymorphism. Each point represents a separate study for the indicated association. $\log [\mathrm{OR}]$ natural logarithm of odds ratio. Horizontal line indicates the effect size.

Inclusion and exclusion criteria. All the studies included in the meta-analysis were required to meet the following criteria: i) case-control studies; ii) evaluation of the association between FAS/FASL polymorphisms and cervical cancer; iii) sufficient data for the estimation of an odds ratio (OR) with $95 \%$ confidence interval (CI). The exclusion criteria were: i) duplicate data; ii) no controls; iii) no sufficient data were reported and; iv) abstract, comment, review and editorial.

Data extraction. Two investigators independently extracted the data and reached consensus on all items. For each eligible study, the following were extracted: the first authors' name, the year of publication, ethnicity (including Caucasian, Asian, patients of African descent and Mixed) of the study population, sources of controls (population- or hospital-based), sample size of cases and controls, genotyping method, distributions of every genotype and evidence of Hardy-Weinberg equilibrium (HWE).

Statistical analysis. ORs with 95\% CIs were calculated to assess the strength of the association between FAS/FASL polymorphisms and cervical cancer risk. To estimate associations with cervical cancer risk, various genotypic models were explored, including the following models: i) additive (minor allele vs. major allele); ii) codominant (heterozygous vs. common homozygous carriers and rare homozygous vs. common homozygous carriers); iii) dominant (rare allele carriers vs. common homozygous carriers); iv) recessive (rare homozygous carriers vs. common allele carriers). Subgroup analyses were performed by ethnicity and source of controls.

Heterogeneity among studies was assessed by the $\chi^{2}$-based Q-statistic (23). Heterogeneity was considered significant for $\mathrm{P}<0.10$. When significant heterogeneity was detected, the random-effects model (DerSimonian and Laird method) (23) was used, otherwise the fixed effects model (Mantel-Haenszel method) (24) was selected. The goodness-of-fit $\chi^{2}$ test was used to assess the deviation from HWE in controls, with statistical significance defined as $\mathrm{P}<0.05$ (25).

Publication bias was evaluated using Begg's funnel plot (26) and Egger's linear regression test (27). Analyses were performed using the software Stata version 12.0 (Stata Corporation, College Station, TX, USA). $\mathrm{P}<0.05$ was considered to indicate a statistically significant difference and all the P-values were two sided.

\section{Results}

Eligible studies. Based on a literature search and selection, a total of 10 publications (11-19,22) comparing the FAS/ FASL polymorphism and cervical cancer susceptibility were identified. Among these studies, 1 study was in Chinese (22) and 1 study contained data on two different ethnicities (18). As a result, 11 case-control studies were included in this meta-analysis. Ten studies on FAS-670A/G polymorphism, five on FAS-1377G/A and six studies on FASL-844T/C polymorphisms met the inclusion criteria (Table I). The genotype distribution in the controls of the studies was consistent with HWE, with the exception of 2 studies for FAS-670 A/G polymorphism $(17,18)$ and 1 study for FASL-844 T/C polymorphism (11) (Table I).

Analysis for FAS-670 A/G polymorphism. A total of 2,127 cases and 2,408 controls were identified for the analysis on FAS-670 A/G polymorphism and cervical cancer risk. The overall result suggested no statistically significant association of this polymorphism with cervical cancer susceptibility (for G vs. A: $\mathrm{OR}=1.016 ; 95 \% \mathrm{CI}, 0.898-1.149 ; \mathrm{P}=0.028$ for heterogeneity; for GG vs. AA: OR=0.944; 95\% CI, 0.729-1.222; $\mathrm{P}=0.058$ for heterogeneity; for $\mathrm{GA}$ vs. $\mathrm{AA}$ : $\mathrm{OR}=1.034 ; 95 \% \mathrm{CI}$, 0.768-1.393; $\mathrm{P}=0.000$ for heterogeneity; for $\mathrm{GG}+\mathrm{GA}$ vs. AA: $\mathrm{OR}=1.043 ; 95 \% \mathrm{CI}, 0.797-1.366 ; \mathrm{P}=0.000$ for heterogeneity; for GG vs. GA/AA: OR=0.999; 95\% CI, 0.784-1.274; $\mathrm{P}=0.004$ for heterogeneity; Table II). In the subgroup analyses by ethnicity and source of controls, no significant associations were found for the genetic models (Table II). Following the exclusion of studies deviating from HWE in controls $(17,18)$, the non-associations in the above-mentioned genetic models remained.

Analysis for FAS-1377 G/A polymorphism. A total of 1,172 cases and 1,476 controls were identified for the analysis 


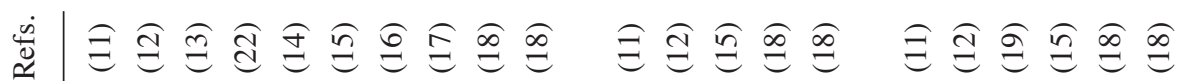

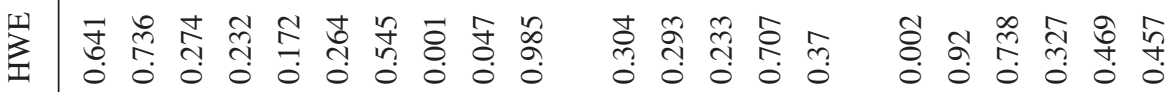

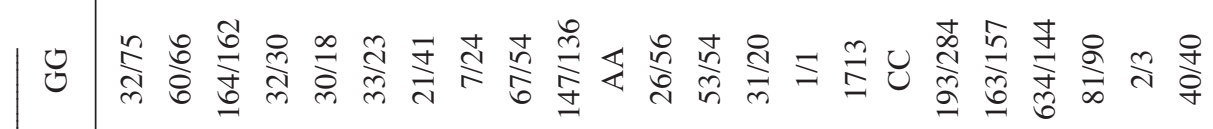

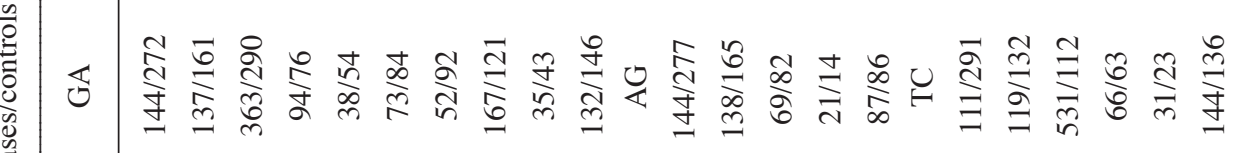

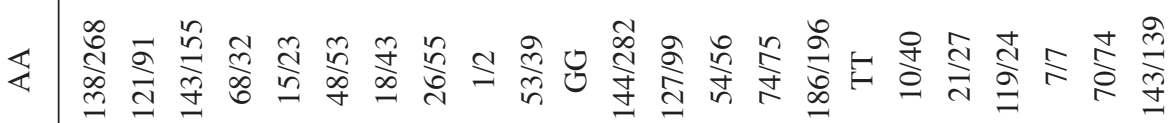

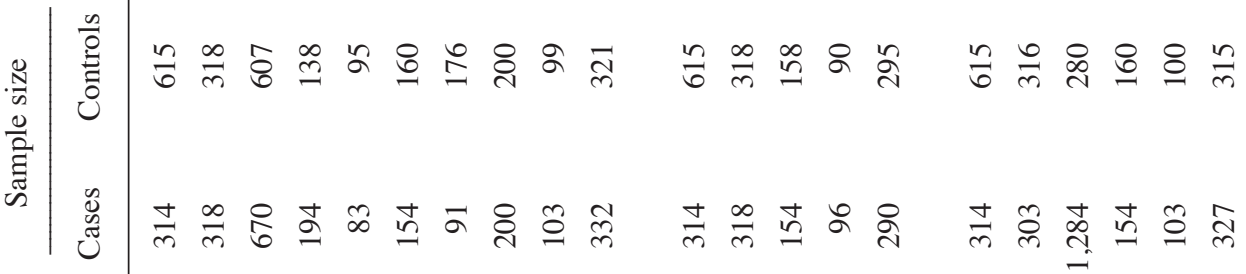

몰

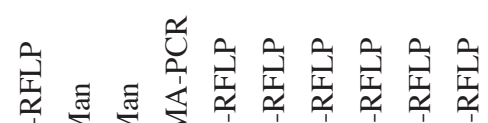

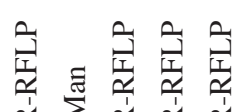

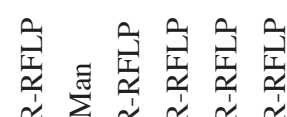

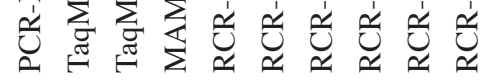

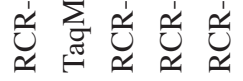

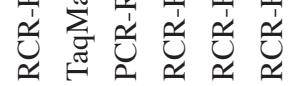

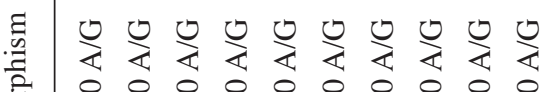

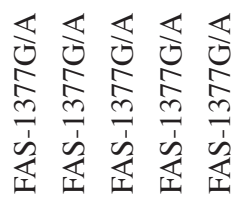

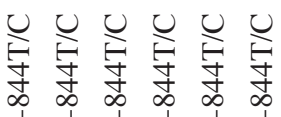

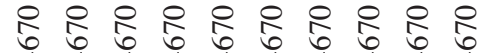

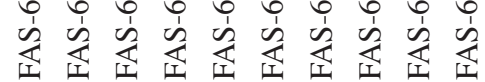

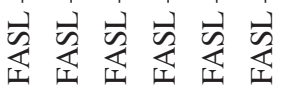

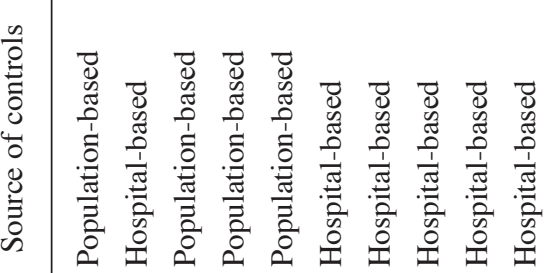

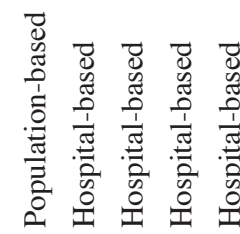

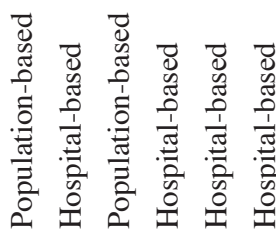

总

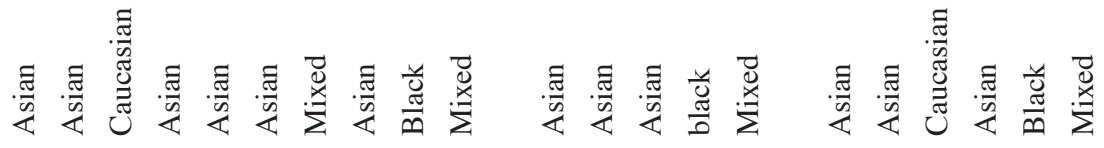

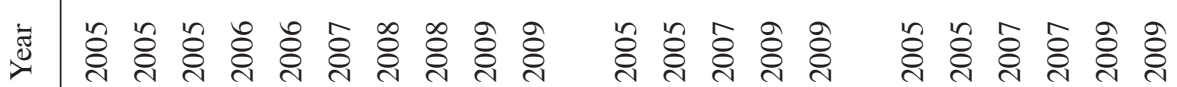

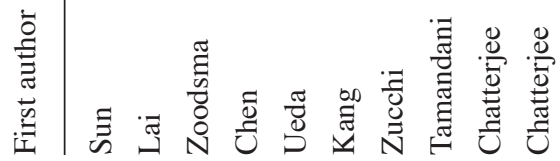

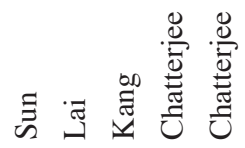

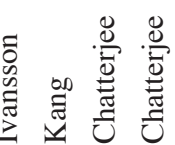

.

.




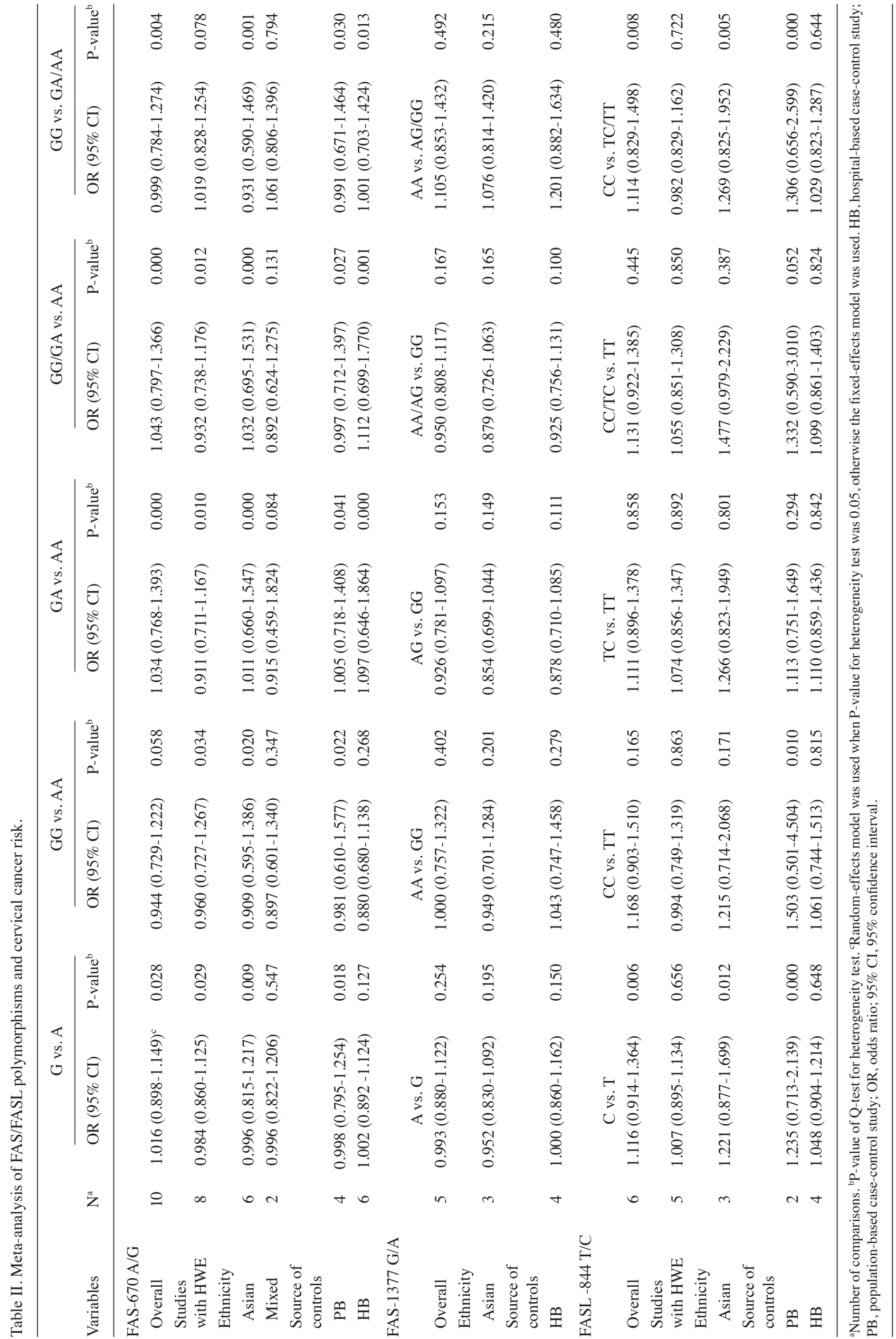


on FAS-1377 G/A polymorphism and cervical cancer risk. The overall result showed that there was no statistically significant association between this polymorphism and cervical cancer susceptibility (Table II). Subsequent subgroup analyses revealed that there was no statistically significant association in each subgroup by ethnicity and source of controls in the genetic models (Table II).

Analysis for FASL-844 T/C polymorphism. A total of 2,485 cases and 1,786 controls were identified for the analysis on FASL-844 T/C polymorphism and cervical cancer risk. The overall result showed that there was no statistically significant association between this polymorphism and cervical cancer susceptibility (Table II). Following exclusion of the study deviating from HWE in controls (11), the results did not alter substantially in any of the genetic models. Subsequent subgroup analyses revealed that there was no statistically significant association in each subgroup by ethnicity and source of controls in the genetic models (Table II).

Publication bias. Both Begg's funnel plot and Egger test were performed to assess the publication bias of the studies. The shape of the funnel plot did not reveal any evidence of obvious asymmetry (Fig. 1). The Egger test was used to provide statistical evidence of funnel plot symmetry. The results again did not suggest any obvious evidence of publication bias for the genetic models (all $\mathrm{P}>0.05$ ).

\section{Discussion}

Genetic variants of the FAS/FASL gene in the etiology of several types of cancer have drawn increasing attention. A number of studies revealed that polymorphic variants of the FAS/FASL polymorphisms were associated with etiology of cervical cancer. However, the results are inconclusive. To gain a better understanding of the association between these polymorphisms and cervical cancer risk, a pooled analysis with a large sample of patients, as well as a subgroup analysis were performed. The aim of our study was to investigate the relationship between FAS/FASL polymorphisms and cervical cancer susceptibility.

In this meta-analysis, we failed to find any association between FAS-670 A/G, FAS-1377 G/A and FASL-844 T/C polymorphisms and cervical cancer risk in the overall analysis. In the subgroup analysis, no association was identified between the three polymorphisms and cervical cancer. These findings indicate that the three polymorphisms may not be risk factors for the development of cervical cancer.

Apoptosis is an important regulatory mechanisms occurring in multicellular organisms that is involved in normal development and tissue homeostasis (28). FAS plays a crucial role in apoptotic signaling and interacts with its natural ligand, FASLG, to initiate the death signal cascade, which results in cell death. Findings of Ueda et al (14) on germ-line polymorphism of Fas promoter -670 demonstrated that the frequency of GG genotype or G allele increased the risk of cervical cancer. Lai et al (12) detected an association between the Fas-670 polymorphism and cervical cancer susceptibility. Zoosdma et al (13) have demonstrated that Fas-670 polymorphism might be involved in the development of adenocarcinoma of the cervix but not in the development of squamous cell carcinoma. Engelmark et al (21), Sun et al (11) and Chatterjee et al (18) did not show a significant association of the FAS/FASL polymorphisms with cervical cancer. Qiu et al (29) indicated that significantly increased risks in FAS-1377 AA carriers were found in the breast cancer subgroup but not in the lung cancer subgroup. Liu et al (30) indicated that the FASL-844C allele was associated with a significantly increased cancer risk overall, but in the stratified analysis by cancer types, significant associations were still not observed in the genetic models. In contrast to these studies, our data failed to reveal significant associations between FAS/FASL polymorphisms and cervical cancer. The cause of these varied results remains unclear, however, discrepancies may exist due to different mechanisms of carcinogenesis in different types of cancer. Cervical cancer is a multi-factorial disease and individual exposures to various environmental factors in combination with genetic susceptibility may have contributed to discrepancies.

However, this study has several limitations. First, significant between-study heterogeneity was detected in some of the comparisons, distorting the findings of the meta-analysis. Second, subgroup analyses concerning other risk factors such as smoking status and HPV infection have not been included in the present study due to insufficient data from the primary literature. Third, further studies estimating the effect of gene-environment interactions may eventually provide an improved, comprehensive understanding of the association between the FAS/FASL polymorphisms and cervical cancer risk. Fourth, relatively small samples of subpopulation may render the interpretation of negative results difficult, requiring more studies with a larger sample size to elucidate those effects. Fifth, the meta-analysis remains a retrospective study that is subject to the methodological deficiencies of the included studies.

In conclusion, our meta-analysis suggests that FAS-670 A/G, FAS-1377 G/A and FASL-844 T/C polymorphisms were not associated with cervical cancer risk. However, large sample studies including different ethnic groups with a careful matching between cases and controls are necessary to confirm our findings.

\section{Acknowledgements}

This study was granted by Anhui Provincial Science and Technology Agency Foundation of China (nos. 11070403061 and 09020303042).

\section{References}

1. Jemal A, Bray F, Center MM, Ferlay J, Ward E and Forman D: Global cancer statistics. CA Cancer J Clin 61: 69-90, 2011.

2. Wallin KL, Wiklund F, Luostarinen T, et al: A population-based prospective study of Chlamydia trachomatis infection and cervical carcinoma. Int J Cancer 101: 371-374, 2002.

3. Schiffman M, Castle PE, Jeronimo J, Rodriguez AC and Wacholder S: Human papillomavirus and cervical cancer. Lancet 370: 890-907, 2007.

4. zur Hausen H: Papillomaviruses and cancer: from basic studies to clinical application. Nat Rev Cancer 2: 342-350, 2002.

5. Andera L: Signaling activated by the death receptors of the TNFR family. Biomed Pap Med Fac Univ Palacky Olomouc Czech Repub 153: 173-180, 2009. 
6. Kim R, Emi M, Tanabe K, Uchida Y and Toge T: The role of Fas ligand and transforming growth factor beta in tumor progression: molecular mechanisms of immune privilege via Fas-mediated apoptosis and potential targets for cancer therapy. Cancer 100: 2281-2291, 2004.

7. Suda T, Takahashi T, Golstein P and Nagata S: Molecular cloning and expression of the Fas ligand, a novel member of the tumor necrosis factor family. Cell 75: 1169-1178, 1993.

8. Huang QR, Morris D and Manolios N: Identification and characterization of polymorphisms in the promoter region of the human Apo-1/Fas (CD95) gene. Mol Immunol 34: 577-582, 1997.

9. Sibley K, Rollinson S, Allan JM, et al: Functional FAS promoter polymorphisms are associated with increased risk of acute myeloid leukemia. Cancer Res 63: 4327-4330, 2003.

10. Wu J, Metz C, Xu X, et al: A novel polymorphic CAAT/enhancerbinding protein beta element in the FasL gene promoter alters Fas ligand expression: a candidate background gene in African American systemic lupus erythematosus patients. J Immuno 170: 132-138, 2003.

11. Sun T, Zhou Y, Li H, et al: FASL -844C polymorphism is associated with increased activation-induced T cell death and risk of cervical cancer. J Exp Med 202: 967-974, 2005.

12. Lai HC, Lin WY, Lin YW, et al: Genetic polymorphisms of FAS and FASL (CD95/CD95L) genes in cervical carcinogenesis: an analysis of haplotype and gene-gene interaction. Gynecol Oncol 99: 113-118, 2005.

13. Zoodsma M, Nolte IM, Schipper M, et al: Interleukin-10 and Fas polymorphisms and susceptibility for (pre)neoplastic cervical disease. Int J Gynecol Cancer 15 (Suppl 3): 282-290, 2005.

14. Ueda M, Terai Y, Kanda K, et al: Fas gene promoter -670 polymorphism in gynecological cancer. Int J Gynecol Cancer 16 (Suppl 1): 179-182, 2006.

15. Kang S, Dong SM, Seo SS, Kim JW and Park SY: FAS-1377 G/A polymorphism and the risk of lymph node metastasis in cervical cancer. Cancer Genet Cytogenet 180: 1-5, 2008.

16. Zucchi F, da Silva ID, Ribalta JC, et al: Fas/CD95 promoter polymorphism gene and its relationship with cervical carcinoma. Eur J Gynaecol Oncol 30: 142-144, 2009.

17. Kordi Tamandani DM, Sobti RC and Shekari M: Association of Fas-670 gene polymorphism with risk of cervical cancer in North Indian population. Clin Exp Obstet Gynecol 35: 183-186, 2008.
18. Chatterjee K, Engelmark M, Gyllensten U, et al: Fas and FasL gene polymorphisms are not associated with cervical cancer but differ among Black and Mixed-ancestry South Africans. BMC Research Notes 2: 238, 2009.

19. Ivansson EL, Gustavsson IM, Magnusson JJ, et al: Variants of chemokine receptor 2 and interleukin 4 receptor, but not interleukin 10 or Fas ligand, increase risk of cervical cancer. Int J Cancer 121: 2451-2457, 2007.

20. Ueda M, Hung YC, Terai Y, et al: Fas gene promoter -670 polymorphism $(\mathrm{A} / \mathrm{G})$ is associated with cervical carcinogenesis. Gynecol Oncol 98: 129-133, 2005.

21. Engelmark MT, Renkema KY and Gyllensten UB: No evidence of the involvement of the Fas-670 promoter polymorphism in cervical cancer in situ. Int J Cancer 112: 1084-1085, 2004.

22. Chen YQ, Lu LG, Tian QF, et al: The research of Fas-670 polymorphism and cervical cancer susceptibility. Natl Med J China 86: 2792-2794, 2006.

23. DerSimonian R and Laird N: Meta-analysis in clinical trials. Control Clin Trials 7: 177-188, 1986.

24. Mantel N and Haenszel W: Statistical aspects of the analysis of data from retrospective studies of disease. J Natl Cancer Inst 22: 719-748, 1959.

25. Haber M: Exact significance levels of goodness-of-fit tests for the Hardy-Weinberg equilibrium. Hum Hered 31: 161-166, 1981.

26. Begg CB and Mazumdar M: Operating characteristics of a rank correlation test for publication bias. Biometrics 50: 1088-1101, 1994.

27. Egger M, Davey Smith G, Schneider M and Minder C: Bias in meta-analysis detected by a simple, graphical test. BMJ 315: 629-634, 1997.

28. Reed JC: Mechanisms of apoptosis. Am J Pathol 157: 1415-1430, 2000.

29. Qiu LX, Shi J, Yuan H, et al: FAS -1,377 G/A polymorphism is associated with cancer susceptibility: evidence from 10,564 cases and 12,075 controls. Hum Gen 125: 431-435, 2009.

30. Liu Y, Wen Q-J, Yin Y, et al: FASLG polymorphism is associated with cancer risk. Eur J Cancer 45: 2574-2578, 2009. 